\title{
Assessment for applicability of the "tangent technique" in X-ray small-angle scattering
}

\author{
A. V. Smirnov, V. Yu. Kalyakin, B. A. Fedorov \\ ITMO University, 49, Kronverkskiy, St. Petersburg, 197101, Russia \\ borfedorov1@gmail.com,smirnav_2@mail.ru, slav.yrich@gmail.com
}

PACS 61.05.cf

DOI 10.17586/2220-8054-2016-7-6-1002-1009

\begin{abstract}
A number of simple model systems are used to examine the applicability of the "tangent technique", employed in the small-angle X-ray scattering for estimating the particle size distribution function, as well as to ascertain the relative contributions of the scattering intensity by differently sized particles to the total scattering intensity. The undertaken analysis has shown that, even in the most favorable case-an ensemble of two groups of different-size particles-the "tangent technique" cannot be used either to find the particle size proper, or to ascertain the relative contributions of individual groups to the total scattering intensity.
\end{abstract}

Keywords: small-angle X-ray scattering, size distribution of nanoparticles, Guinier plot.

Received: 27 July 2016

Revised: 4 August 2016

\section{Introduction}

The numerous methods used to process experimental curves of X-ray small-angle scattering with the view to derive information on the structure of scattering objects include the "tangent technique" [1,2]. This seemed simple and convenient enough for estimating the size distribution of globular nanoparticles as well as for ascertaining the relative contributions of particles of each size to the total scattering intensity. At the same time, the technique, as even its authors remarked, is rather limited in its scope. Though it was proposed as early as the 1950's and has since been repeatedly used in the small-angle scattering [3,4], no one has tried to evaluate the errors arising from its application. Moreover, no one has raised the issue whether its use is altogether possible. Will errors drive the required parameters beyond the reasonable values even in the case of the simplest scattering systems and in the scattering angle region, which is most suitable for application of the technique?

The present work attempts to answer the questions by undertaking a detailed analysis of applicability of the "tangent technique" in the cases of both model systems and a globular protein mixture.

\section{Description of the technique}

As is known, the intensity $I(q)$ of the small-angle X-ray scattering by a particle, which comprises $N$ different scattering centers (atoms) and is chaotically oriented relative to the primary X-ray beam, can be represented with Debye formula [5]:

$$
I(q)=\sum_{i, j=1}^{N} f_{i}(q) f_{j}(q) \frac{\sin \left(q r_{i j}\right)}{q r_{i j}}
$$

where $r_{i j}$ is the distance between the $i$-th and $j$-th atoms, $q=(4 \pi / \lambda) \sin (\theta / 2)$ is the magnitude of the reciprocal space vector ( $\lambda$ is the X-ray wavelength, $\theta$ is the scattering angle), $f_{i}(q)$ is the scattering factor of the $i$-th atom. If $q$ is small, $f_{i}(q)$ is weakly dependent on $q$ and $f_{i}(q)=f_{i}(0)=f_{i}$, the number of electrons in the $i$-th atom.

Expansion of Eq. (1) into a series gives:

$$
I(q)=\sum_{i, j=1}^{N} f_{i} f_{j}\left(1-\frac{q^{2} r_{i j}^{2}}{6}+\ldots\right)=A^{2}\left(1-\frac{q^{2} R_{g}^{2}}{3}+\ldots\right),
$$

where $R_{g}^{2}=\frac{\sum_{i, j=1}^{N} f_{i} f_{j} r_{i j}^{2}}{2 \sum_{i, j=1}^{N} f_{i} f_{j}}$ is the squared electron radius of gyration of a particle and $A^{2}=\sum_{i, j=1}^{N} f_{i} f_{j}$ is the squared number of electrons in a particle. 
The series expansion of function $A^{2} \exp \left(-\frac{1}{3} R_{g}^{2} q^{2}\right)$ is easily seen to have first two terms coincident with Eq. (2). This property underlies the Guinier technique (e.g. [6-9]): plotting the scattering intensity as ln $I(q)$ vs. $q^{2}$ in the region of small angles yields a straight line, its slope allowing one to find $R_{g}^{2}$, i. e., the particle size.

Consider a system, consisting of differently sized particles, and assume the scattering intensity for each particle to follow the exponential law. Then, the scattering intensity for the entire system - without interference between individual particles - is presentable in the form:

$$
\tilde{I}(q)=\sum_{i=1}^{N} K_{i} A_{i}^{2} \exp \left(-\frac{1}{3} R_{g i}^{2} q^{2}\right),
$$

where $N$ is the number of groups of particles with radii of gyration $R_{g i}$ and $K_{i}$ is the particle count in each group. Let the summation be from the largest $(i=1)$ to the smallest particle $(i=N)$.

If the number of groups is rather small and radii of gyration $R_{g i}$ vary from group to group appreciably, the "tangent technique" was believed to be fully justified, with inclusion of the following stages:

1) Plotting $\ln \tilde{I}(q)$ vs. $q^{2}$.

2) Drawing a tangent to the curve at the largest values of $q$, but in the scattering angle region, where exponential approximation Eq. (3) is valid for all groups of scattering particles.

3) Finding squared radius of gyration $\tilde{R}_{g N}^{2}$ for the smallest particles by the tangent slope according to Eq. (2) and value $\tilde{K}_{N} A_{N}^{2}$ by the intercept of the tangent on axis $\ln \tilde{I}(q)$. If $A_{N}$ is known (roughly, the value $A_{N}$ is one-half of the molecular mass of the particle), one also finds $\tilde{K}_{N}$, the particle count in group $N$.

4) Subtracting function $\tilde{K}_{N} A_{N}^{2} \exp \left(-\frac{1}{3} \tilde{R}_{g N}^{2} q^{2}\right)$ with already known values of $\tilde{K}_{N}$ and $\tilde{R}_{g N}^{2}$ from the experimental intensity Eq. (3). One finds scattering intensity $\tilde{I}(q)$ by the remaining particles and passes on to Operation 1) with substituting group $N$ with group $N-1$.

Upon completion of the said cycle, the following sought parameters are calculated: radii of gyration $\tilde{R}_{g i}$ (sizes) of all particles included in the scattering system and the relative particle count in each group. One can also plot $\tilde{K}_{i}$ vs. $\tilde{R}_{g i}$ (which is sometimes done [4]) to evaluate the particle size distribution, which is dedicated to other methods in the small-angle scattering $[10,11]$.

Despite the deceptive simplicity of the technique and the value of the derived information, the application of the technique gives rise to several somewhat complex questions:

a) How is the angle region to be chosen for the condition of Eq. (2) to hold?

b) What are the grounds to believe that the scattering intensity in the vicinity of drawing each tangent is governed by but one group of particles, while other groups make no contributions whatsoever to the scattering curve in this angular region?

c) How is, when drawing tangents, the straight-line segment of the scattering curve to be distinguished from the curvilinear one, and in the presence of the unavoidable experimental error at that?

d) How is the number of groups with different values of $R_{g}$ to be found under the conditions of an actual experiment?

There is, apparently, no general answer to the questions, so the present work examines a number of specific different-size systems to assess the applicability of the technique.

\section{Analysis of the technique applicability}

Simplify the problem statement as much as possible. To begin with, assume only two groups of differently sized particles $(N=2)$. This is evidently the most favorable model. If the technique proves inapplicable even for this model, there is no sense to increase the number of groups. Next, an interval of scattering angles is to be selected for drawing the tangents and, accordingly, to specify the particle sizes in each. The basic condition is that the intensity, plotted in Guinier coordinates for particles of both groups, had the shape of a straight line in the selected scattering angle interval. Of importance is also for the angular interval to be available experimentally. In terms of the magnitude of reciprocal space vector, the interval is defined from $q_{\min }=0.01 \mathrm{~nm}^{-1}$ to $q_{\max }=0.14 \mathrm{~nm}^{-1}$ (hereinafter, a bounded interval), which corresponds (at X-ray wavelength $\lambda_{C u K \alpha}=0.154 \mathrm{~nm}$ ) to scattering angles from $\theta_{\min }=2.5 \cdot 10^{-4}$ rad to $\theta_{\max }=3.4 \cdot 10^{-3} \mathrm{rad}$ and, respectively, to Bragg spacings from $d_{\max }=300 \mathrm{~nm}$ to $d_{\text {min }}=23 \mathrm{~nm}$. Particle sizes (radii of gyration $R_{g}$ ) were chosen as $R_{g 1}=15.56 \mathrm{~nm}, R_{g 2}=9.21 \mathrm{~nm}$. It has been shown for particles of such sizes that the required condition of straightness of Guinier plots in the said scattering angle interval holds reasonably well. Let $K_{1}$ and $K_{2}$ be the initial particle counts in each group, and $\tilde{K}_{1}$ and $\tilde{K}_{2}$ be those found by the "tangent technique". 
For all particle groups considered under the said conditions, we calculated scattering intensities, constructed Guinier plots, evaluated the quantities $\tilde{R}_{g 1}, \tilde{R}_{g 2}, \tilde{K}_{1}, \tilde{K}_{2}$ by the "tangent technique" in a wide variation range of $K_{2} / K_{1}$, and estimated the errors of the quantities as compared to their true values. The results are presented in Tables 1-4. In each table, column 1 is the selected value of $K_{2} / K_{1}$; columns 2 and 3 are the calculated relative errors of quantities $\tilde{R}_{g 1}$ and $\tilde{R}_{g 2}\left(\delta R_{g i}=\left(\tilde{R}_{g i}-R_{g i}\right) / R_{g i}\right)$; columns 4 and 5 are the calculated relative errors of quantities $\tilde{K}_{1}$ and $\tilde{K}_{2}\left(\delta K_{i}=\left(\tilde{K}_{i}-K_{i}\right) / K_{i}\right)$.

Hypothetical particles with scattering intensity defined by Eq. (3) at $N=2$. The scattering curve for each particle is shaped in Guinier coordinates as a straight line, yet the dependence of $\ln \left[K_{1} A_{1}^{2} \exp \left(-\frac{1}{3} R_{g 1}^{2} q^{2}\right)+\right.$ $\left.K_{2} A_{2}^{2} \exp \left(-\frac{1}{3} R_{g 2}^{2} q^{2}\right)\right]$ on $q^{2}$ is no longer a straight line. In a wide scattering angle interval $\left(q_{\min }=0.01 \mathrm{~nm}^{-1}\right.$ to $\left.q_{\max }=0.4 \mathrm{~nm}^{-1}\right)$, of course, the effects related to the influence of different groups on the total scattering curve are separable (Fig. 1), and the "tangent technique" yields reasonable values for radii of gyration and particle counts in each group (Table 1). However, the effects cannot be separated in the said bounded scattering angle interval.

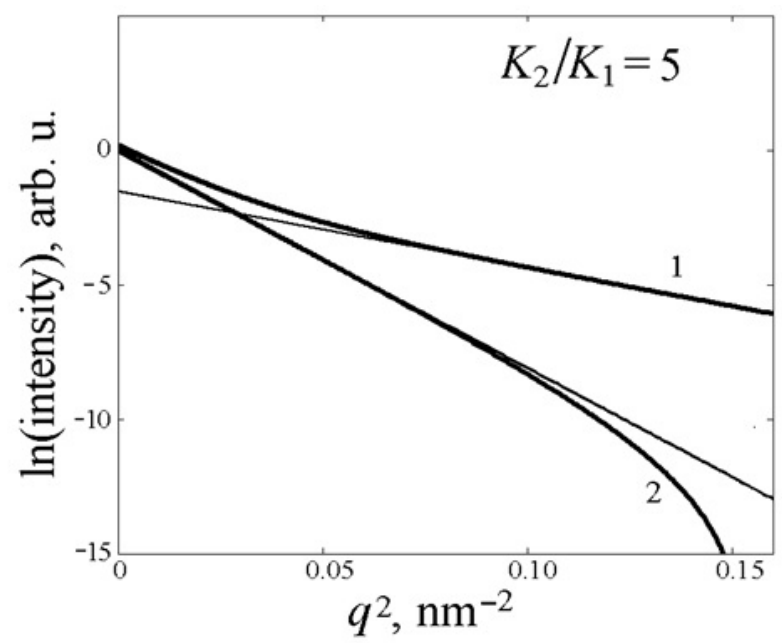

FIG. 1. Hypothetical particles. Curve 1 is the scattering intensity found according to Eq. (3) at $N=2$; curve 2 is the difference scattering curve between intensity of Eq. (3) and the intensity of $\tilde{K}_{2} A_{2}^{2} \exp \left(-\frac{1}{3} \tilde{R}_{g 2}^{2} q^{2}\right)$. All curves are plotted in Guinier coordinates. Straight lines are tangents to Curves 1 and 2. Wide scattering angle interval. See details in the text

TABLE 1. Hypothetical particles. Wide scattering angle interval $\left(q_{\min }=0.01 \mathrm{~nm}^{-1}\right.$ to $q_{\max }=$ $\left.0.4 \mathrm{~nm}^{-1}\right)$. Relative errors of radii of gyration $\tilde{R}_{g 1}, \tilde{R}_{g 2}$ and weighting factors $\tilde{K}_{1}, \tilde{K}_{2}$ at different ratios $K_{2} / K_{1}$. See details in the text

\begin{tabular}{|c|c|c|c|c|}
\hline$K_{2} / K_{1}$ & $\delta R_{g 1}, \%$ & $\delta R_{g 2}, \%$ & $\delta K_{1}, \%$ & $\delta K_{2}, \%$ \\
\hline 5 & 0.08 & 0.13 & -0.3 & 1.3 \\
\hline 10 & 0.08 & 0.06 & -0.3 & 0.6 \\
\hline 20 & 0.08 & 0.03 & -0.3 & 0.3 \\
\hline 50 & 0.08 & 0.013 & -0.3 & 0.13 \\
\hline 100 & 0.08 & $6 \cdot 10^{-3}$ & -0.3 & $6 \cdot 10^{-2}$ \\
\hline 150 & 0.08 & $4 \cdot 10^{-3}$ & -0.3 & $4 \cdot 10^{-2}$ \\
\hline
\end{tabular}

Table 2 presents the results of applying the technique in a bounded interval at different ratios $K_{2} / K_{1}$. As seen, in the case of the number of small particles much greater than that of larger ones $\left(K_{2} / K_{1}=150\right.$ and more), the contribution of the latter tells little on the scattering curve. Hence, quantities $\tilde{R}_{g 2}$ and $\tilde{K}_{2}$ are evaluated comparatively well, but the calculation of quantities $\tilde{R}_{g 1}$ and $\tilde{K}_{1}$ yields unacceptable errors. At a smaller $K_{2} / K_{1}$ 
TABLE 2. Hypothetical particles. Bounded scattering angle interval $\left(q_{\min }=0.01 \mathrm{~nm}^{-1}\right.$ to $q_{\max }=0.14 \mathrm{~nm}^{-1}$ ). Relative errors of radii of gyration $\tilde{R}_{g 1}, \tilde{R}_{g 2}$ and weighting factors $\tilde{K}_{1}, \tilde{K}_{2}$ at different ratios $K_{2} / K_{1}$. See details in the text

\begin{tabular}{|c|c|c|c|c|}
\hline$K_{2} / K_{1}$ & $\delta R_{g 1}, \%$ & $\delta R_{g 2}, \%$ & $\delta K_{1}, \%$ & $\delta K_{2}, \%$ \\
\hline 5 & 47 & 48 & -90 & 419 \\
\hline 10 & 46 & 38 & -86 & 200 \\
\hline 20 & 44 & 26 & -82 & 96 \\
\hline 50 & 43 & 14 & -79 & 37 \\
\hline 100 & 43 & 8 & -78 & 18 \\
\hline 150 & 43 & 5 & -78 & 12 \\
\hline
\end{tabular}

ratio, likely unacceptable errors emerge in evaluation of all four quantities in question. This can now be explained by the effect of the scattering intensity of larger particles on the total curve in the entire bounded interval. As a consequence, quantities $\tilde{R}_{g 2}$ and $\tilde{K}_{2}$ are evaluated with great errors, while the errors in the evaluation of quantities $\tilde{R}_{g 1}$ and $\tilde{K}_{1}$, naturally, grow significantly.

Homogeneous ellipsoids of revolution and spheres. As previously shown [12], the scattering intensity by the $i$-th homogeneous ellipsoid of revolution with electron density $\rho$ is defined by expression $A_{i}^{2} I_{i}^{e l}(q)$, where $A_{i}=\rho V_{i}, V=\frac{4}{3} \pi a^{2} b$ is the volume of the ellipsoid ( $b$ is the semi-major axis, $a$ is the semi-minor axis),

$$
I_{i}^{e l}(q)=\int_{0}^{1} \Phi^{2}\left(q \sqrt{5 R_{g i}^{2}\left(1+x^{2}\left(\nu^{2}-1\right)\right) /\left(2+\nu^{2}\right)}\right) d x
$$

is its formfactor $\left(I_{i}^{e l}(0)=1\right), \Phi(t)=3 \frac{\sin t-t \cos t}{t^{3}}$, and $\nu=b / a$. Radius of gyration $R_{g}$ is related to semi-axes $a$ and $b$ by $R_{g}=\sqrt{\left(2 a^{2}+b^{2}\right) / 5}$.

The total scattering intensity for two groups of ellipsoids is representable as:

$$
I^{e l}(q)=K_{1} A_{1}^{2} I_{1}^{e l}(q)+K_{2} A_{2}^{2} I_{2}^{e l}(q),
$$

where, as before, $K_{1}$ and $K_{2}$ are the particle counts in the first and second groups, respectively. We chose anisotropy $\nu=2$ the same for ellipsoids of both groups, since the calculations show the straight-line segment in Guinier plotting to prove to be the most extended at this anisotropy.

Table 3 presents the calculation results. They are close to those obtained for hypothetical particles in a bounded interval of scattering angles. And while the relative errors for quantities $\tilde{R}_{g 2}$ and $\tilde{K}_{2}$ are comparatively small in the most favorable case $\left(K_{2} / K_{1}=150\right)$, those for $\tilde{R}_{g 1}$ and $\tilde{K}_{1}$ are as high as tens of percent.

TABLE 3. Ellipsoids of revolution. Relative errors of radii of gyration $\tilde{R}_{g 1}, \tilde{R}_{g 2}$ and weighting factors $\tilde{K}_{1}, \tilde{K}_{2}$ at different ratios $K_{2} / K_{1}$. Bounded scattering angle interval $\left(q_{\min }=0.01 \mathrm{~nm}^{-1}\right.$ to $\left.q_{\max }=0.14 \mathrm{~nm}^{-1}\right)$. See details in the text

\begin{tabular}{|c|c|c|c|c|}
\hline$K_{2} / K_{1}$ & $\delta R_{g 1}, \%$ & $\delta R_{g 2}, \%$ & $\delta K_{1}, \%$ & $\delta K_{2}, \%$ \\
\hline 5 & 47 & 44 & -87 & 404 \\
\hline 10 & 46 & 34 & -83 & 194 \\
\hline 20 & 45 & 24 & -80 & 93 \\
\hline 50 & 43 & 12 & -77 & 36 \\
\hline 100 & 43 & 6.3 & -75 & 17 \\
\hline 150 & 42 & 4.1 & -73 & 11 \\
\hline
\end{tabular}


The model of a scattering system is very often taken to be spheres. As follows from Eq. (4), the form-factor of the scattering intensity for spheres $(\nu=1)$ is:

$$
I_{i}^{s p h}(q)=\Phi^{2}\left(\sqrt{\frac{5}{3}} q R_{g i}\right),
$$

and the total intensity for two groups of spheres is:

$$
I^{s p h}(q)=K_{1} A_{1}^{2} I_{1}^{s p h}(q)+K_{2} A_{2}^{2} I_{2}^{s p h}(q) .
$$

The results of the corresponding calculation are given in Table 4. They demonstrate that the errors in evaluating all four quantities prove to be unacceptable. In the most favorable case (as before, at $K_{2} / K_{1}=150$ ), the errors in evaluating quantities $\tilde{R}_{g 1}$ and $\tilde{K}_{1}$ are as high as tens of percent, whereas quantity $\tilde{R}_{g 1}$ at $K_{2} / K_{1}=5$ cannot be calculated at all, for the slope of the corresponding tangent happens to be positive.

TABLE 4. Spheres. Relative errors of radii of gyration $\tilde{R}_{g 1}, \tilde{R}_{g 2}$ and weighting factors $\tilde{K}_{1}, \tilde{K}_{2}$ $K_{2} / K_{1}$. Bounded scattering angle interval $\left(q_{\min }=0.01 \mathrm{~nm}^{-1}\right.$ to $\left.q_{\max }=0.14 \mathrm{~nm}^{-1}\right)$. See details in the text

\begin{tabular}{|c|c|c|c|c|}
\hline$K_{2} / K_{1}$ & $\delta R_{g 1}, \%$ & $\delta R_{g 2}, \%$ & $\delta K_{1}, \%$ & $\delta K_{2}, \%$ \\
\hline 5 & unevaluable & 64 & unevaluable & 482 \\
\hline 10 & 26 & 49 & -96 & 223 \\
\hline 20 & 37 & 35 & -91 & 105 \\
\hline 50 & 40 & 20 & -88 & 41 \\
\hline 100 & 42 & 13 & -91 & 21 \\
\hline 150 & 49 & 10 & -94 & 15 \\
\hline
\end{tabular}

Globular proteins. The procedure for calculating the intensity of X-ray scattering by globular proteins in a solution, which was developed by one of the authors jointly with others [13-15], is applicable in the case when the coordinates of all atoms of the protein under study are known. Were we concerned with the scattering intensity of such protein in vacuum, the problem would not be difficult: it would suffice to use Debye formula Eq. (1). However, as was shown experimentally as early as in works [16,17], the presence of the solvent has an effect upon the entire scattering curve of a protein.

Briefly, the method of allowing for the solvent effect can be described as follows. If a protein contains $N$ nonhydrogen atoms with coordinates $r_{i}$ and scattering factor $f_{i}(q)$, its scattering intensity can be found in accordance with the expression:

$$
I(q)=\langle I(\mathbf{q})\rangle=\left\langle\left|\sum_{j=1}^{N} f_{j}(q) \exp (i \mathbf{q r})-\rho_{s} \Psi(\mathbf{q})\right|^{2}\right\rangle,
$$

the averaging is done over all possible orientations of the protein relative to the primary beam, $\rho_{s}$ is the solvent electron density, and function:

$$
\Psi(\mathbf{q})=\int_{V} \exp (i \mathbf{q r}) d r
$$

is the amplitude of scattering by that homogeneous volume of a protein particle, which is inaccessible to the solvent molecules. The calculation of the amplitude is a major difficulty. We developed a "cube method" [18], which bases on filling the entire particle volume, inaccessible to the solvent, with small cubes (with edges $0.0279 \mathrm{~nm}$ long); their dense packing ensures the homogeneity of density inside the molecule. Thus, the calculation of the amplitude of scattering in Eq. (9) reduces to finding the amplitude of scattering by a system of cubes (parallelepipeds). Next, scattering intensity $I(\mathbf{q})$ is evaluated at point $\mathbf{q}$ of the reciprocal space, and intensity $I(q)$ is found by averaging $I(\mathbf{q})$ over a sphere of radius $q=|\mathbf{q}|$ of the space. The procedure of the said averaging is described in [19].

Consider a mixture of two globular proteins with atom coordinates given in the PDB: human hemoglobin (http://files.rcsb.org/view/4ESA.pdb) and hen egg-white lysozyme (http://files.rcsb.org/view/6LYZ.pdb). Their radii of gyration are specified as $R_{g 1}=15.56 \mathrm{~nm}$ and $R_{g 2}=9.21 \mathrm{~nm}$, respectively. For aqueous solution of the two proteins, scattering intensities were calculated and total curves were derived at different ratios $K_{2} / K_{1}$. Table 5 gives the values of all four estimated parameters in a wide variation range of $K_{2} / K_{1}$. 
TABLE 5. Globular proteins in solution: human hemoglobin and hen egg-white lysozyme. Relative errors of radii of gyration $\tilde{R}_{g 1}, \tilde{R}_{g 2}$ and weighting factors $\tilde{K}_{1}, \tilde{K}_{2}$ at different ratios $K_{2} / K_{1}$. Bounded scattering angle interval $\left(q_{\min }=0.01 \mathrm{~nm}^{-1}\right.$ to $\left.q_{\max }=0.14 \mathrm{~nm}^{-1}\right)$. See details in the text

\begin{tabular}{|c|c|c|c|c|}
\hline$K_{2} / K_{1}$ & $\delta R_{g 1}, \%$ & $\delta R_{g 2}, \%$ & $\delta K_{1}, \%$ & $\delta K_{2}, \%$ \\
\hline 5 & 18 & 24 & -77 & 56 \\
\hline 10 & 19 & 15 & -75 & 27 \\
\hline 20 & 16 & 8.8 & -75 & 14 \\
\hline 50 & 3.5 & 4.8 & -79 & 5.8 \\
\hline 100 & -61 & 3.3 & -87 & 3.2 \\
\hline 150 & unevaluable & 2.8 & unevaluable & 2.3 \\
\hline
\end{tabular}

As is readily seen, when the number of lysozyme molecules is predominant in the solution, the lysozyme radius of gyration and the relative number of molecules are evaluated within $10 \%$ accuracy down to $K_{2} / K_{1}=50$. However, the hemoglobin scattering intensity in the region of very small angles is distorted by the scattering intensity of all lysozyme molecules, and, as a consequence, the errors in evaluation of quantities $\tilde{R}_{g 1}$ and $\tilde{K}_{1}$ prove to be too great or the quantities are unevaluable altogether. At smaller ratios $K_{2} / K_{1}$, the evaluation errors of all four quantities are unacceptable. This result, likewise to the earlier-treated case of hypothetical particles, is explained by the effect of the scattering intensity of large particles upon the total curve now in the entire bounded interval, in particular, upon its right-hand part, which is used to calculate $\tilde{R}_{g 2}$ and $\tilde{K}_{2}$.

\section{Discussion of results}

The main conclusion, following from the above examination, is that the "tangent technique" is, from the practical viewpoint, inapplicable to any of the analyzed scattering systems. This is demonstrated by the data given in all five tables.

To exemplify the conclusion graphically, consider the scattering intensity for two groups of ellipsoids of revolution in Guinier coordinates at a great value of ratio $K_{2} / K_{1}=150$ (Fig. 2a) and a small value of $K_{2} / K_{1}=5$ (Fig. 2b). Both figures show four scattering curves: 1 and 2 are those for the first and second groups of particles; 3 is the total scattering intensity, corresponding to Eq. (5); 4 is the difference scattering curve between intensities of Eq. (5) and $\tilde{K}_{2} A_{2}^{2} \exp \left(-\frac{1}{3} \tilde{R}_{g 2}^{2} q^{2}\right)$, where $\tilde{R}_{g 2}$ is the radius of gyration of smaller particle, which was found by the slope of the tangent to the curve in the right-hand part of the bounded scattering angle interval.

For larger $K_{2} / K_{1}$ ratio values, the prevailing contribution to the total scattering intensity is seen from Fig. 2a to come from the scattering by small particles; still, the tangents to curves 2 and 3 in the right-hand part of the bounded interval differ a little both in the slope and the intercept on the axis of ordinates. The differences suffice to distort sought quantities $\tilde{R}_{g 1}$ and $\tilde{K}_{1}$ substantially: the slopes of the tangents to curves 1 and 4 in the left-hand part of the bounded interval differ markedly, and the intercepts on the axis of ordinates differ, too. At the same time, quantities $\tilde{R}_{g 2}$ and $\tilde{K}_{2}$ proper are evaluated well enough.

At relatively small values of ratio $K_{2} / K_{1}$ (Fig. 2b), the principal contribution to the total scattering intensity in the entire bounded interval comes from the large particle, so the tangents to curves 2 and 3 differ markedly even in the right-hand part of the interval. The intercepts of the tangents on the axis of ordinates differ markedly, too. In other words, a large error arises in the evaluation of quantities $\tilde{R}_{g 2}$ and $\tilde{K}_{2}$, and, hence, even larger error in the evaluation of quantities $\tilde{R}_{g 1}$ and $\tilde{K}_{1}$.

If the bounded scattering angle interval is extended, the technique is seen from Fig. 1 to be completely applicable, but solely for hypothetical particles, which are non-existent in nature. Indeed, there is no realistic particle, for which the scattering intensity would follow the exponential law in a wide angular region. Under any other dependence, even at the first stage of applying the technique (that is, in evaluation of $\tilde{R}_{g 2}$ by the tangent slope), an appreciable error arises, which grows sharply in calculation of other quantities at hand.

It may seem that if the particle sizes $\left(R_{g 1}\right.$ and $\left.R_{g 2}\right)$ differ markedly, say, by an order of magnitude, the "tangent technique" is completely applicable. However, as follows from Eq. (3), the contributions to the total scattering intensity by large and small particles (at their equal shares in terms of numbers) differ $\left(R_{g 1} / R_{g 2}\right)^{6}$ times. In this case, for their contributions to the scattering intensity to be comparable, one should take at least 

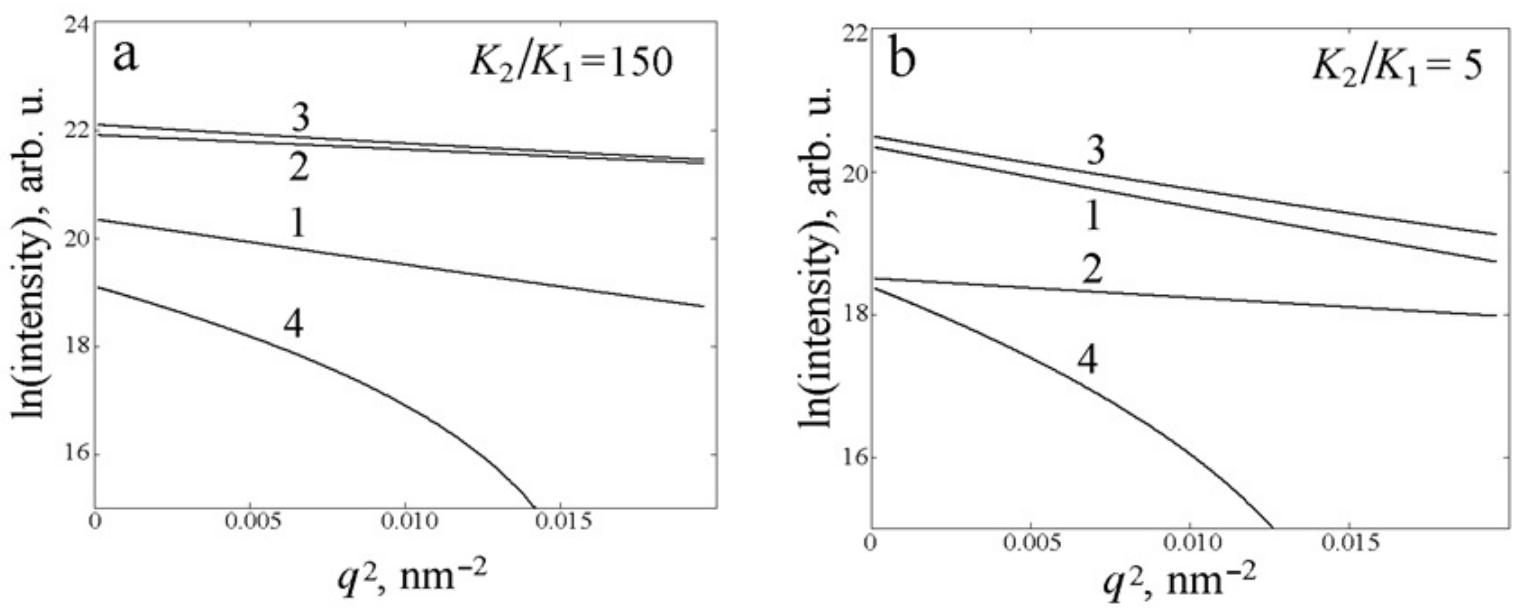

FIG. 2. Scattering intensities for two groups of ellipsoids of revolution. 1 and 2 are scattering curves for the first $\left(R_{g 1}=15.56 \mathrm{~nm}\right)$ and second $\left(R_{g 2}=9.21 \mathrm{~nm}\right)$ groups of ellipsoids, respectively; (3) is the total scattering intensity, found according to Eq. (5); 4 is the difference scattering curve between intensities of Eq. (5) and $\tilde{K}_{2} A_{2}^{2} \exp \left(-\frac{1}{3} \tilde{R}_{g 2}^{2} q^{2}\right)$. All curves are plotted in Guinier coordinates. See details in the text

million more small particles, which renders the technique absolutely impractical and the calculation of quantities $\tilde{R}_{g 1}$ and $\tilde{K}_{1}$ fraught with even greater errors.

\section{Conclusion}

The results of the present work can be deemed negative. The grand total is that the "tangent technique" cannot be used for any different-size scattering particle groups either to find the particle size proper, or to ascertain the relative contributions of individual groups to the total scattering intensity. The statement is true for two groups of particles and even more so for a greater number of groups. The ratio of particle sizes in different groups does not affect the general negative result.

\section{Acknowledgements}

The authors are grateful to ITMO university student N. S. Bondarev for his assistance in calculations.

\section{References}

[1] Porai-Koshits E.A. Small Angle X-Ray Diffuse Scattering. Uspekhi fizicheskikh nauk, 1949, 39(4), P. $573-611$.

[2] Kitaigorodskii A.I. X-ray Analysis of Fine-Grained Crystalline and Amorphous Bodies, Gostekhizdat, Moscow-Leningrad, 1952 , 588 p.

[3] Jellinek M.H., Soloman Ernest, Fankuchen I. Measurement and Analysis of Small-Angle X-Ray Scattering. Ind. Eng. Chem. Anal. Ed., 1946, 18(3), P. 172-175.

[4] Kyutt R.N., Smorgonskaya É.A., Danishevskii A.M., Gordeev S.K., Grechinskaya A.V. Structural studies of nanoporous carbon produced from silicon carbide. Physics of the Solid State, 1999, 41(5), P. 808-810.

[5] Debye P. Zerstreuung von Röntgenstrahlen. Annalen der Physik, 1915, 351(6), P. 809-823.

[6] Guinier A., Fournet G. Small-Angle Scattering of X-Rays, New-York, John Wiley, 1955, 268 p.

[7] Porod G. General Theory in Small-Angle X-Ray Scattering, edited by O. Glatter \& O. Kratky. London, Academic Press, 1982 , P. 17-52.

[8] Almjasheva O.V., Fedorov B.A., Smirnov A.V., Gusarov V.V. Size, morphology and structure of the particles of zirconia nanopowder obtained under hydrothermal conditions. Nanosystems: Physics, Chemistry, Mathematics, 2010, 1(1), P. $26-36$.

[9] Smirnov A.V., Deryabin I.N., Fedorov B.A. Small-angle scattering: the Guinier technique underestimates the size of hard globular particles due to the structure-factor effect. J. Appl. Cryst., 2015, 48, P. 1089-1093.

[10] Letcher J.H., Schmidt P.W. Small Angle X Ray Scattering Determination of Particle Diameter Distributions in Polydisperse Suspensions of Spherical Particles. J. Appl. Cryst., 1966, 37, P. 649-655.

[11] Kuchko A.V., Smirnov A.V. The computation of the nanoparticles volume distribution function and the specific surface area based on the small-angle X-ray scattering indicatrix by the method of the statistical regularization. Nanosystems: Physics, Chemistry, Mathematics, 2012, 3(3), P. 76-91.

[12] Porod G. Abhängigkeit der Röntgen-Kleinwinkelstreuung von Form und Grösse der kolloiden Teilchen in verdünnten Systemen. IV. Acta Physica Austriaca, 1948, 2, P. 255-292.

[13] Fedorov B.A., Ptitsyn O.B. and Voronin L.A. X-Ray Diffuse Scattering by Proteins in Solution. Consideration of Solvent Influence. J. Appl. Cryst., 1974, 7, P. 181. 
[14] Denesyuk A.I. Large-Angle Diffuse Scattering, a New Method for Investigating Changes in the Conformation of Globular Proteins in Solutions, J. Appl. Cryst., 1978, 11, P. 473-477.

[15] Pavlov M.Yu., Fedorov B.A. The method of calculating the surface and the volume of the protein in a solvent. Biofizika, 1982, 27(4), P. 609-613.

[16] Stuhrmann H.B. Ein neues Verfahren zur Bestimmung der Oberflächenform und der inneren Struktur von gelösten globularen Proteinen aus Röntgenkleinwinkelmessungen. Zeitschr. Physik. Chem. Neue Folge, 1970, 72, P. 185-198.

[17] Ibel K., Stuhrmann H.B. Comparison of neutron and X-ray scattering of dilute myoglobin solutions. J. Mol. Biol., 1975, 93, P. 255-265.

[18] Pavlov M.Yu., Fedorov B.A. Improved technique for calculating X-ray scattering intensity of biopolymers in solution: Evaluation of the form, volume, and surface of a particle. Biopolymers, 1983, 22, P. 1507-1522.

[19] Knyazev S.N., Kalyakin V.Yu., Deryabin I.N., Fedorov B.A., Smirnov A.V., Stepanov E.O., Porozov Yu.B. Prediction of Protein Conformational Mobility with Validation Using Small Angle X-Ray Scattering. Biophysics, 2015, 60(6), P. 886-892. 